\title{
CircLMNBI promotes colorectal cancer by regulating cell proliferation, apoptosis and epithelial-mesenchymal transition
}

This article was published in the following Dove Press journal: OncoTargets and Therapy

\section{Chunping $\mathrm{He}$ \\ Chao Huang \\ Rui Zhou \\ Honggang Yu}

Department of Gastroenterology, Renmin Hospital of Wuhan University, Wuhan 430060, Hubei Province, People's Republic of China
Correspondence: Honggang Yu Department of Gastroenterology, Renmin Hospital of Wuhan University, No. 99 Zhangzhidong Road, Wuhan 430060, Hubei Province, People's Republic of China Tel +86 I $387 \quad$ I28 I899

Email doctoryuhg@163.com
Objective: The aberrant expression of circular RNAs (circRNAs) is a frequent occurrence in various cancers. However, the functions and roles played by most circRNAs in colorectal cancer (CRC) remain largely unknown.

Materials and methods: Levels of circLMNB1 expression were evaluated by qRT-PCR and FISH assays. The influence of circLMNB1 knockdown on LoVo and HCT116 cell proliferation, cycling, apoptosis, migration, and invasion were assessed by the CCK-8, assay, Edu assay, flow cytometry, Hoechst staining, and the Transwell assay, respectively. The relative levels of EMT- and apoptosis-related proteins were determined by Western blotting.

Results: CircLMNB1 expression was significantly upregulated in CRC tissues and cells. Knockdown of circLMNB1 by siRNA in LoVo cells suppressed cell proliferation, migration and invasion, and facilitated cell cycle arrest and apoptosis In addition, we proved that knockdown of circLMNB1 upregulated E-cadherin, Bax and caspase-3 expression, and downregulated MMP2, MMP-9, and N-cadherin expression in LoVo cells. Further results showed that overexpression of circLMNB1 enhanced the malignant characteristics of HCT116 cells. Conclusion: Our findings revealed that blocking of circLMNB1 could inhibit CRC development, and help to explain the underlying mechanism by which circLMNB1 knockdown inhibits the metastasis of CRC. Finally, this study suggests circLMNB1 as a novel biomarker for CRC. Keywords: circLMNB1, colorectal cancer, epithelial-mesenchymal transition, matrix metalloproteinase-2/9

\section{Introduction}

Colorectal cancer (CRC) is one of the common digestive system malignancies worldwide, and a serious threat to people's physical and mental health. ${ }^{1}$ Although patients with early-stage CRC have high cure and 5-year survival rates, most patients with CRC are initially diagnosed at an advanced stage of their disease due to a lack of effective biomarkers for early-stage $\mathrm{CRC}$, and the 5-year survival rate of those patients is only $10-15 \% .^{2}$ Therefore, early diagnosis plays an essential role in reducing the $\mathrm{CRC}$ mortality rate, and research on biomarkers is of great significance for the early diagnosis, individual treatment, and detection of recurrent CRC.

Circular RNAs (circRNAs) comprise a new class of non-coding RNAs (ncRNAs) that are produced by the head-to-tail splicing of several exons of a target gene $^{3}$ and are widely expressed in eukaryotes. ${ }^{4,5}$ Due to their specific method of formation, circRNAs usually have a covalently closed loop structure without a $5^{\prime}$ cap and a $3^{\prime}$ tail. $^{6}$ Based on their source, circRNAs can be divided into two types: 
exonic circRNAs (exclusively containing exon sequences), which are mainly located in the cytoplasm, and exon intronic circRNAs (retaining intron sequences), which are most commonly found in the cell nucleus. ${ }^{4}$ Numerous studies have shown that the circRNA expression profiles in cancer cell lines and tissues are different from those in corresponding normal cell lines and tissues. ${ }^{7,8}$ In addition, recent studies have confirmed that circRNAs participate in various tumor cell-associated physiological processes, including proliferation, differentiation, cycling, apoptosis, migration, and invasion. ${ }^{9}$ CircRNA microarray studies have also shown that circRNA expression profiles in samples of CRC tissue differ from those in samples of corresponding normal tissue. ${ }^{10-13}$ circRNAs can be coding from $L M N B 1$, and in this research, hsa_circ_0127801 was identified as candidate in this study. However, the molecular mechanisms and physiological functions of circLMNB1 have not been reported in the literature.

It is well recognized that tumor metastasis is a complex processes that involves multiple stages, pathways, and genetic changes. ${ }^{13}$ A growing body of evidence indicates that the epithelial-mesenchymal transition (EMT) is a major mechanism of cancer cell invasion and metastasis, and produces a phenotypic conversion. ${ }^{14}$ The EMT is a process by which epithelial cells lose their epithelial characteristics and change into interstitial cells under physiological or pathological conditions. ${ }^{15}$ During EMT, differentiated epithelial cells lose their apical-basal polarity and epithelial adhesion (E-cadherin positive), and acquire a myofibroblastic phenotype ( $\alpha$-smooth muscle actin, vimentin, and $\mathrm{N}$-cadherin positive), which is accompanied by enhanced cell migration and invasion capabilities. ${ }^{16}$ Therefore, we further explored whether circLMNB1 might affect CRC progression by regulating the EMT.

In the present study, we found that circLMNB1 was highly expressed in CRC tissues and cells, and we also investigated the effects of circLMNB1 knockdown on CRC cell proliferation, cell cycle arrest, apoptosis, migration, and invasion capabilities, as well as EMT characteristics. Our results suggest circLMNB1 as a potential biomarker for use in diagnosing CRC.

\section{Materials and methods CRC tissue samples}

Specimens of CRC tissue and adjacent tissue $(5 \mathrm{~cm}$ separation from the lesion area) were obtained from a total 20 CRC patients who underwent surgery at the
Renmin Hospital of Wuhan University between March 2015 and January 2017. The patients had no other types of tumors, had never received chemo- or radiotherapy, and were being treated for the first time. The operations were performed under aseptic conditions, and all patients provided written informed consent. The study protocol was approved by the Ethics Committee Board of Renmin Hospital of Wuhan University, and all procedures were performed in accordance with the Declaration of Helsinki.

\section{Cell culture}

A normal colorectal cell line (FHC) was purchased from the Institute of Biochemistry and Cell Biology of the Chinese Academy of Sciences (Shanghai, China), and 5 human CRC cell lines (HT29, LoVo, HCT116, SW480, and RKO) were purchased from Lonza Biologics (Hayward, CA, USA). SW480 cells were cultured in Leibovitz's L-15 medium (Sigma, St. Louis, MO, USA; L1518), HT29 cells were cultured in MCCOYS' 5A medium (Sigma, M9309), and FHC, HCT116, LoVo, and RKO cells were cultured in RPMI 1640 medium (Hyclone, Logan, UT, USA; SH30809.01B). All media were mixed with $10 \%$ fetal bovine serum (FBS, BI, cat. no. 04-001-1A) and antibiotics (100 units $/ \mathrm{mL}$ penicillin and $100 \mathrm{mg} / \mathrm{mL}$ streptomycin). All cells were cultured at $37^{\circ} \mathrm{C}$ in a humidified atmosphere containing $5 \% \mathrm{CO}_{2}$.

\section{Cell transfection}

SiRNA (siRNA: 5'-GTCAGAGACATCTTACGTT-3') targeting circLMNB1 was used to block circLMNB1 expression in CRC cells. LoVo cells were seeded into 6-well plates at a density of $1 \times 10^{6}$ cells per well and transfected with a negative control (NC) or circLMNB1 siRNA (circLMNB1) by use of Lipofectamine 3000 (Invitrogen, Carlsbad, CA, USA) according to the manufacturer's instructions.

\section{RNA extraction and quantitative real- time PCR (qRT-PCR) assay}

TRIzol reagent (\#9109, Takara, Japan) was used to extract the total RNA from CRC and para-carcinoma tissues, as well as treated and untreated CRC cell lines. A Bestar ${ }^{\mathrm{TM}}$ qPCR RT kit (\#2220, DBI Bioscience, China) was used to reverse transcribe a $5 \mu \mathrm{g}$ sample of total RNA according to the manufacturer's instructions. The qRT-PCR assay was performed by using SYBRs GREEN PCR Master Mix (Applied Biosystems, Warrington, UK) on an ABI 7000 RT-PCR system according to the protocol provided by the manufacturer. The level of circLMNB1 expression was normalized to that of GAPDH (an 
endogenous housekeeping gene) and calculated using the $2^{-\triangle \triangle \mathrm{Ct}}$ method. ${ }^{17}$ The sequences of primers used were GAPDH: forward, 5'-CCA GCC GAG CCA CATCGC TC$3^{\prime}$ and reverse, 5'-ATG AGC CCC AGC CTT CTC CAT-3'; circLMNB1: forward, 5'-GCCAAAATTGAATGCTGTCC-3' and reverse, 5'-TGAGATAGCCCAGCAATCCT-3'. The amplified products were then sequenced by Sangon Biotech (Sangon Biotech, Shanghai, China).

\section{Construction of the recombinant carrier}

Circ_LMNB1 was cloned by using the forward primer (AGC TGG CCC TTC TCA AGA CGG ATC C) and reverse primer (ACT ATA CTG GTT GAG CTC TCC ACT CGA ATT C) with BamHI and EcoRI restriction enzyme cutting sites, and then inserted into a pcircRNA2.2 vector (BersinBio, Guangzhou, China). Next, the recombinant carrier was transfected into competent cells, followed by monoclonal sequencing detection.

\section{Western blot assay}

The total proteins were isolated by using lysis buffer containing a protease inhibitor cocktail (P8340; Sigma). The protein concentration in each sample was evaluated by using a BCA protein assay kit (Thermo Fisher Scientific, Waltham, MA, USA). A $30 \mu \mathrm{g}$ sample of total protein from each sample was separated by $10 \%$ SDS-PAGE, and the separated protein bands were transferred onto polyvinylidene fluoride (PVDF) membranes (Millipore, Bedford, MA, USA), which were then blocked with skimmed milk (5\%) for 2 hrs at room temperature. Next, the membranes were incubated overnight at $4{ }^{\circ} \mathrm{C}$ with primary antibodies, followed by incubation with the secondary antibodies (HRP linked, Abcam, Cambridge, UK, ab205718, 1:2,000) at room temperature for $2 \mathrm{hrs}$. The blots were exposed by using ECL detection reagent (EMD Millipore) and analyzed by using ImageJ version 1.48 software (National Institutes of Health, Bethesda, MD, USA). The primary antibodies used in this study were anti-E-cadherin (Abcam, ab76319, 1:1,000), antiMMP2 (Abcam, ab2462, 1:1,000), anti-MMP9 (Abcam, ab228402, 1:1,000), anti-N-cadherin (Abcam, ab76057, 1:1,000), anti-Bax (Abcam, ab53154, 1:1,000), anti-Caspase-3 (Abcam, ab214430, 1:1,000), and anti-GAPDH (Abcam, ab9485, 1:2,000).

\section{Fluorescence in situ hybridization (FISH) assay}

To determine the circLMNB1 fusion status in LoVo cells, cells or samples of slide-mounted tissue were incubated with a circLMNB1 probe at $73 \pm 1{ }^{\circ} \mathrm{C}$ for 5 mins. Next, the specimens were washed and sequentially dehydrated in $70 \%$, $85 \%$, and $100 \%$ pre-cooled ethanol for 3 mins; after which, hybridization was allowed to occur overnight at $45-50{ }^{\circ} \mathrm{C}$. After washing, images were obtained under a fluorescence microscope (Eclipse E600; Nikon Corporation, Tokyo, Japan). The sections were stained with 4' 6-diamidino-2phenylindole (DAPI, Cat\#H-1200; Vector Laboratories) for 45 mins in the dark.

\section{CCK-8 assay}

The CCK-8 assay was used to evaluate cell proliferation. Cells were seeded in 96-well plates at a density of $2 \times 10^{3}$ cells per well, and then cultured for 24,48 , or 72 hrs. CCK-8 solution (10 $\mu \mathrm{L}$ ) (Obio Technology, Shanghai, China) was added to each well at the designated time point, and the plates were then incubated at $37^{\circ} \mathrm{C}$ for an additional $2 \mathrm{hrs}$. The optical density (OD) of each well at $450 \mathrm{~nm}$ was recorded.

\section{EdU assay}

A Cell-Light 5-ethynyl-2-deoxyuridine (EdU) DNA Cell Proliferation Kit (RiboBio, Guangzhou, China) was utilized to determine cell viability. Briefly, treated LoVo cells were seeded in 24-well plates at a density of $1 \times 10^{5}$ cells per well, and then exposed to $30 \mu \mathrm{M} \mathrm{EdU}$ at $37{ }^{\circ} \mathrm{C}$ for $2 \mathrm{hrs}$. Next, the cells were fixed with $4 \%$ formaldehyde (cat. no. 153814; Biosharp, Wuhan, China) at room temperature for 30 mins, and then permeabilized by exposure to $0.5 \%$ Triton X-100 for 10 mins; after which, they were washed and stained with DAPI. After staining, cell fluorescence was measured by flow cytometry.

\section{Cell cycle and apoptosis analysis}

For cell cycle assays, the treated LoVo cells were harvested, fixed in ice-cold $70 \%$ ethanol, and then stored at $4{ }^{\circ} \mathrm{C}$ overnight. Next, the fixed cells were resuspended in PBS, and then treated with $10 \mu \mathrm{g} / \mathrm{mL}$ propidium iodide (PI; Sigma, Aldrich) and $100 \mathrm{mg} / \mathrm{L}$ RNase for $30 \mathrm{mins}$ in the dark. An Annexin V-FITC/PI apoptosis detection kit (BestBio, Shanghai, China) was used for apoptosis assays. Briefly, treated LoVo cells $\left(1 \times 10^{6}\right.$ cells $\left./ \mathrm{mL}\right)$ were suspended in $100 \mu \mathrm{L}$ of $1 \times$ binding buffer and then double stained with Annexin V-FITC/PI for 15 mins according to the manufacturer's instructions. Cell cycle phase and apoptosis were evaluated with a FACS Calibur Flow Cytometer (BD Biosciences, San Jose, CA, USA), and the results were analyzed by using ModFit LT 2.0 software. 


\section{Hoechst 33258 staining}

A Hoechst 33258 staining kit was used according to manufacturer's instructions. Cells were plated into a 96-well plate, and then collected for experiments $24 \mathrm{~h}$ after transfection. Briefly, the cells were covered with $100 \mu \mathrm{L}$ of Hoechst 33258 working reagent, and then incubated for $25 \mathrm{~min}$ at $37{ }^{\circ} \mathrm{C}$. Afterwards, the cells were washed twice with PBS, and then observed under a laser scanning confocal microscope.

\section{Migration and invasion assays}

Cell migration and invasion assays were conducted by using 24-well Transwell plates with an $8 \mu \mathrm{m}$ pore size (BD Biosciences, Franklin Lakes, NJ, USA) according to instructions provided by the manufacturer. The cells in each treatment group were collected and diluted to a final concentration of $2 \times 10^{5}$ cells $/ \mathrm{mL}$, and then starved for $12 \mathrm{~h}$. Next, $200 \mu \mathrm{L}$ of cell suspension was added to each upper chamber that contained $0.2 \%$ FBS, and $600 \mu \mathrm{L}$ of culture medium containing $20 \%$ FBS was added to each lower chamber. After $24 \mathrm{hrs}$, the migrated cells were fixed in 4\% paraformaldehyde (cat. No. P6148; Sigma) and stained with crystal violet solution (Cat. No: C-3886, Sigma-Aldrich, St. Louis, MO, USA). The results were observed under a light microscope. For the invasion assay, the upper chambers of each Transwell plate were pretreated with Matrigel (cat\# 356230, BD Biosciences) at $37{ }^{\circ} \mathrm{C}$ for 30 mins.

\section{Statistical analysis}

All statistical data were analyzed using GraphPad Prism 7 software (La Jolla, CA, USA), and results are presented as the mean \pm standard deviation (SD). Differences between groups were analyzed by one-way analysis of variance (ANOVA). Each experiment was performed 3 times, and a $P$-value $<0.05$ was considered to be statistically significant.

\section{Results}

\section{CircLMNBI was highly expressed in CRC tissues and cells}

The qRT-PCR assay was used to investigate the levels of circLMNB1 expression in CRC cells and tissues. The results showed that among 20 matched pairs of CRC tumor tissues and para-carcinoma tissues, circLMNB1 was more highly expressed in the CRC tissues than in the paracarcinoma tissues $(P<0.05$, Figure $1 \mathrm{~A})$. We next determined the levels of circLMNB1 expression in human fetal colon (FHC) cells and 5 CRC cell lines (HT29, LoVo, HCT116,
SW480, and RKO). The results showed that when compared with circLMNB1 expression in FHC cells, circLMNB1 expression was significantly increased in the CRC cell lines; with the highest levels of expression being found in LoVo cells, followed by RKO, HCT116, SW480, FHC, and HT29 cells $\left({ }^{*} P<0.05,{ }^{* *} P<0.01,{ }^{* * *} P<0.001\right.$, Figure 1B). Therefore, LoVo cells were selected as a model in which to explore the molecular mechanism and biological function of circLMNB1. In addition, we utilized agarose electrophoresis after PCR amplification, and Sanger sequencing to confirm the expression and abundance of circLMNB1 in LoVo cells (Figure 1C and D). Results of a FISH assay indicated that circLMNB1 was expressed in the cytoplasm of LoVo cells (Figure 1E).

\section{Knockdown of circLMNBI inhibited proliferation and facilitated cell cycle arrest in LoVo cells}

The frequent upregulation of circLMNB1 in CRC tissues and cells suggested that circLMNB1 might play a significant role in CRC progression. To assess the possible role of circLMNB1 in CRC, we transfected LoVo cells with siRNA against circLMNB1 to knock down endogenous circLMNB1 expression $\left({ }^{*} P<0.01\right.$, Figure $\left.2 \mathrm{~A}\right)$. However, expression of OMNB1 is not mediated by siRNA. CCK-8 and Edu assays were carried out to evaluate the effect of circLMNB1 knockdown on the proliferative ability of LoVo cells. As shown in Figure $2 \mathrm{~B}$ and $\mathrm{C}$, cell viability was significantly decreased in the siRNA group (circLMNB1knockdown LoVo cells) when compared with the NC group $(* * * P<0.001)$. In order to further explore the potential mechanism responsible for reduced cell viability after circLMNB1 knockdown, flow cytometry analyses were performed to determine the cell cycle distributions of cells in the siRNA treatment and NC control groups, respectively. Those results showed that the cell number of G1 phase cells in the siRNA group was much higher than that in the NC group, and the number of S phase cells in the siRNA group was much lower than that in the $\mathrm{NC}$ group (Figure 2D). These findings indicated that knockdown of circLMNB1 suppressed cell proliferation, and promoted cell cycle arrest in LoVo cells.

\section{Knockdown of circLMNBI accelerated cell apoptosis in LoVo cells}

After circLMNB1 knockdown in LoVo cells, a flow cytometry analysis and Hoechst staining were performed to verify the 
A

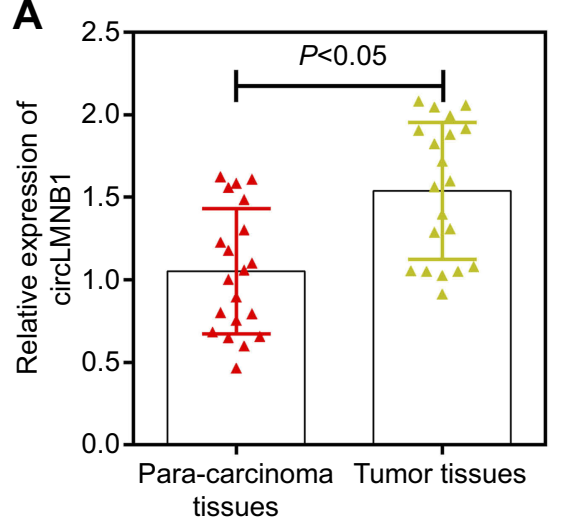

C

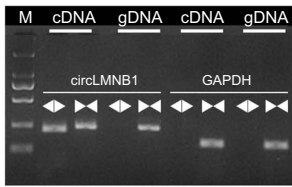

D

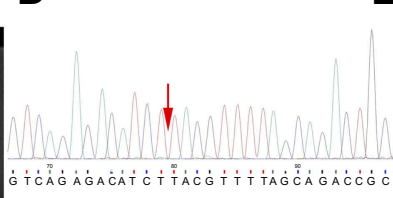

B

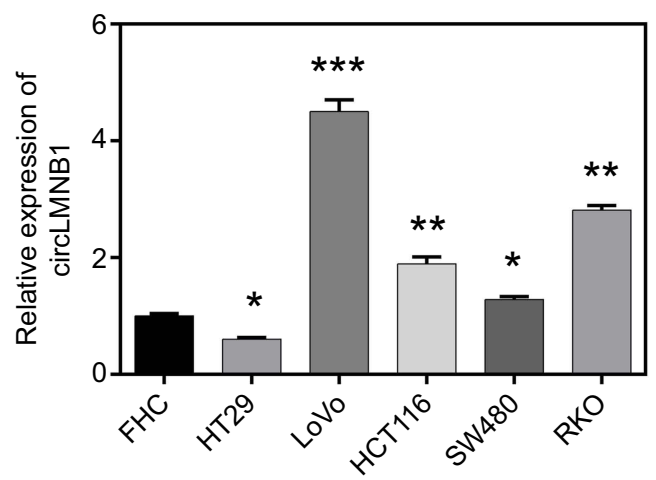

E CircLNMB1
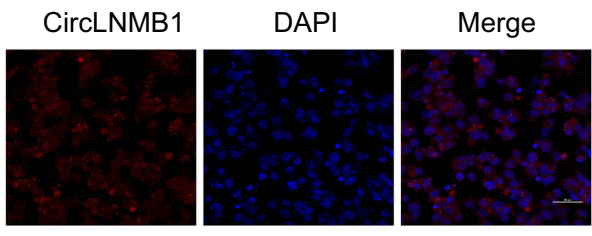

Figure I CircLMNBI was highly expressed in colorectal cancer (CRC) tissues and cells. (A) The level of circLMNBI expression was determined by the qRT-PCR assay in 20 pairs of $C R C$ tissues and para-carcinoma tissues $(P<0.05)$. (B) CircLMNBI expression was assessed by the $q R T-P C R$ assay in human fetal colon (FHC) cells and $C R C$ cell lines (HT29, LoVo, HCTII6, SW480, and RKO), respectively ( $* P<0.05$, $* * P<0.01, * * * P<0.001$ vs FHC group). (C, D) CircLMNBI was validated using PCR (C) and Sanger sequencing (D). (E) The level of circLMNBI expression in LoVo cells was evaluated with the FISH assay. Magnification $\times 200$, scale bar $=50 \mu \mathrm{m}$.

influence of circLMNB1 on the apoptosis capability of LoVo cells. Annexin V FITC/PI staining indicated that the percentages of early and late apoptotic cells in the siRNA group were increased relative to those percentages in the $\mathrm{NC}$ group (Figure 3A). Moreover, Hoechst staining results also showed that knockdown of circLMNB1 promoted CRC cell apoptosis (Figure 3B). Finally, results of Western blotting assays showed that knockdown of circLMNB1 promoted the expression of two pro-apoptotic proteins, Bax and caspase-3 (Figure 3C).

\section{Knockdown of circLMNBI suppressed the migration and invasion of LoVo cells}

The migration and invasion capabilities of LoVo cells with circLMNB1 knockdown were evaluated with the Transwell assay. The results showed that the numbers of migrated and invaded cells were obviously reduced in the siRNA group when compared with those numbers in the NC group, suggesting that circLMNB1 downregulation had inhibited LoVo cell migration and invasion (Figure 4A-C). These findings proved that knockdown of circLMNB1 could inhibit the ability of LoVo cells to metastasize. To further explore the mechanism by which circLMNB1 affects CRC progression, the EMT property of LoVo cells was investigated. In those studies, we examined the expression of EMT-related proteins and apoptosis-related proteins by use of Western blot assays. As shown in Figure 4D, the levels of E-cadherin expression in the siRNA group were higher than those in the NC group, while MMP2, MMP-9, and Ncadherin expression were all decreased in the siRNA group when compared with their expression in the NC group. These data further demonstrated that knockdown of circLMNB1 suppressed EMT and promoted apoptosis.

\section{Overexpression of circLMNBI increased the malignant characteristics of HCTII6 cells}

The CCK8 and EdU staining assays were used to explore the effect of circLMNB1 expression on cancer cell viability. As shown in Figure 5A, transfection of a circLMNB1 plasmid into HCT116 cells forced overexpression of circLMNB1, but had no effect on LMNB1 expression (Figure 5A). The results of subsequent CCK8 and EdU assays showed that overexpression of circLMNB1 significantly promoted cell viability (Figure 5B and $\mathrm{C}$ ).

Transwell assays were performed to investigate how circLMNB1 affects cell mobility and apoptosis. Results showed that cell mobility was enhanced by overexpression of circLMNB1 (Figure 5D and E). Forward scatter results from flow cytometric (FSC) studies indicated that cell apoptosis was inhibited when circLMNB1 was overexpressed in HCT116 cells (Figure 5F). Finally, Western 
A

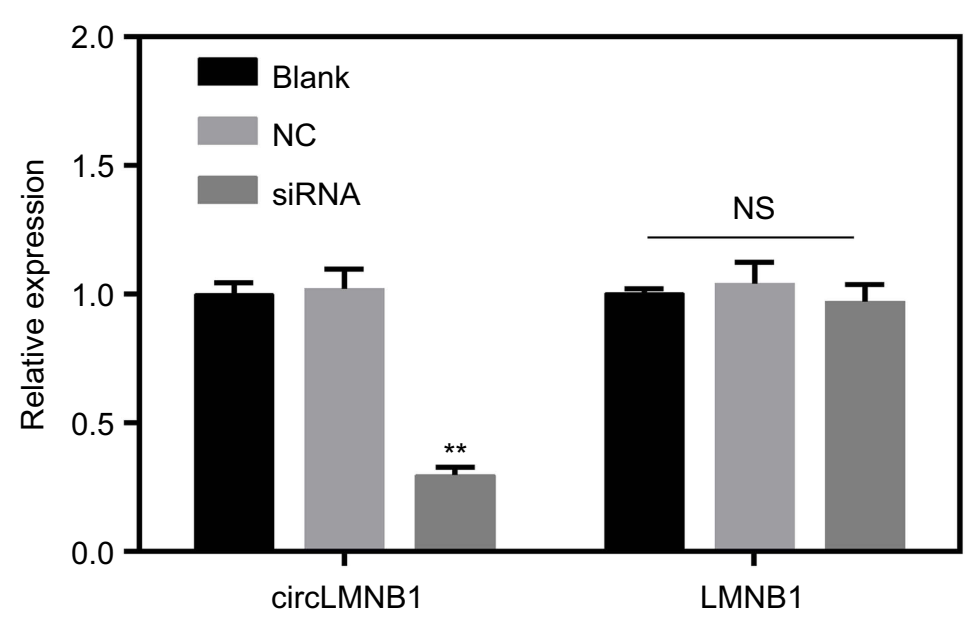

C

Blank

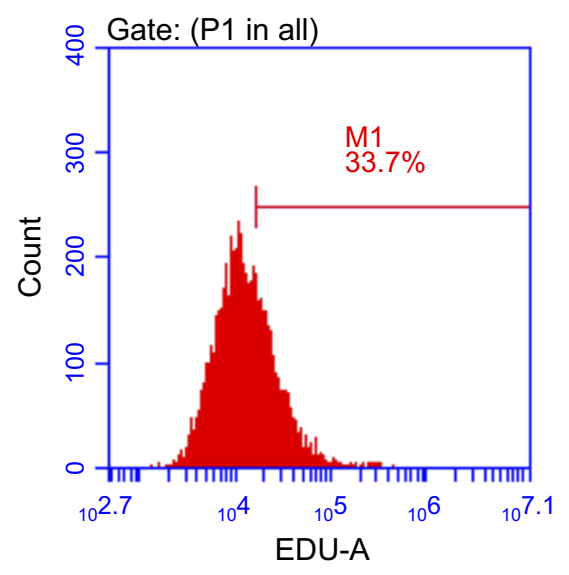

D

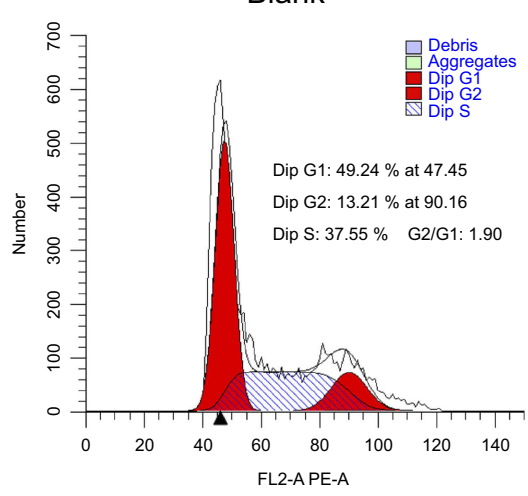

NC
B

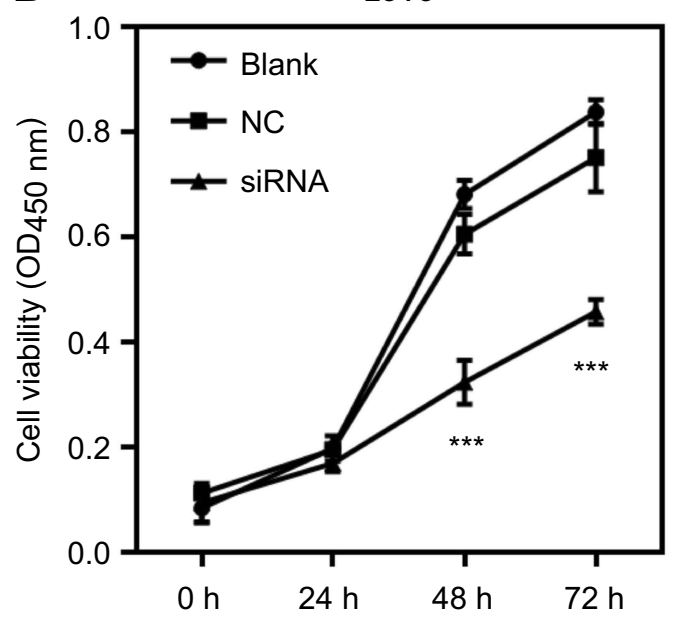

SiRNA
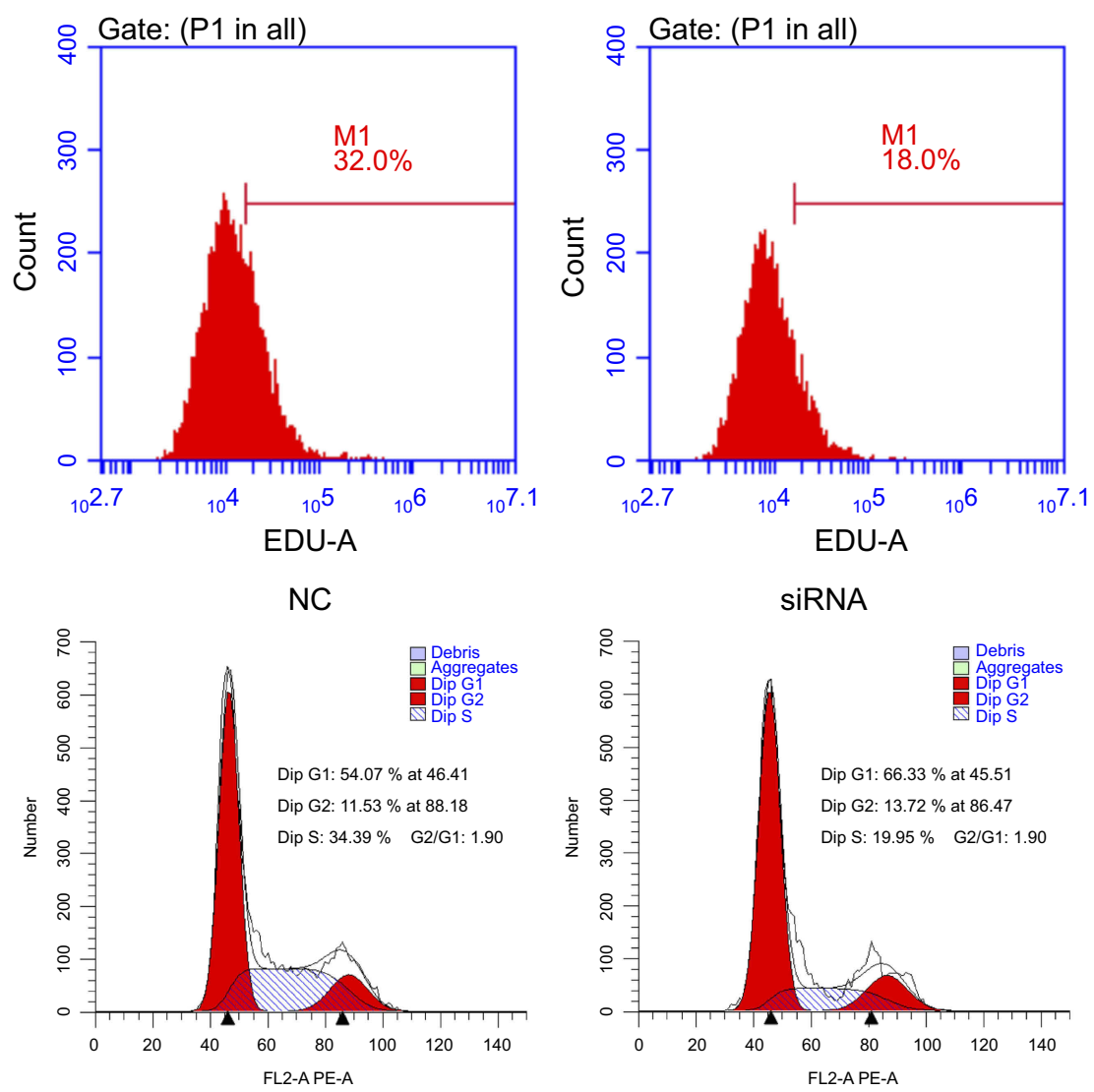

SiRNA

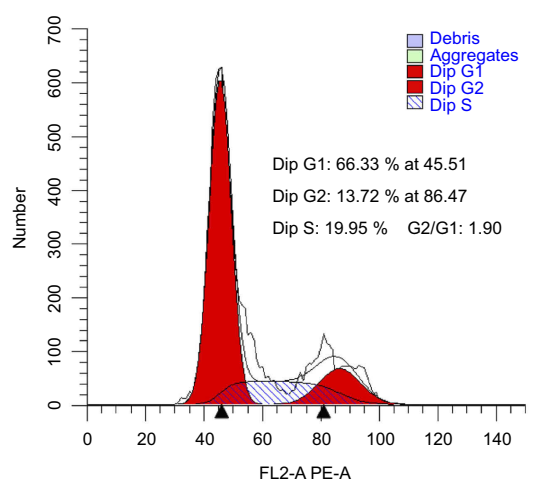

Figure 2 Knockdown of circLMNBI inhibited proliferation, and facilitated cell cycle arrest in LoVo cells. LoVo cells were transfected with NC and circLMNBI siRNA, respectively. (A) Expression of circLMNBI and LMNBI in LoVo cells was evaluated by the qRT-PCR assay after circLMNBI knock-down (**P<0.0I vs NC group). (B) The proliferation of LoVo cells after circLMNBI knock-down was determined by using the CCK-8 assay (***P<0.00I vs NC group). (C) The proliferation of LoVo cells with circLMNBI-knockdown was measured by flow cytometry with Edu staining. (D) The cell cycle distribution of LoVo cells with circLMNBI knockdown was determined by flow cytometry.

blot assays showed that the apoptosis-related proteins Bax and caspase-3 were down-regulated after circLMNB1 overexpression, and metastasis-related proteins were also affected (Figure 5G).

\section{Discussion}

Numerous studies have proven that the expression profiles of non-coding RNAs (ncRNAs), which mainly consist of microRNAs (miRNAs) and long non-coding RNAs 
A

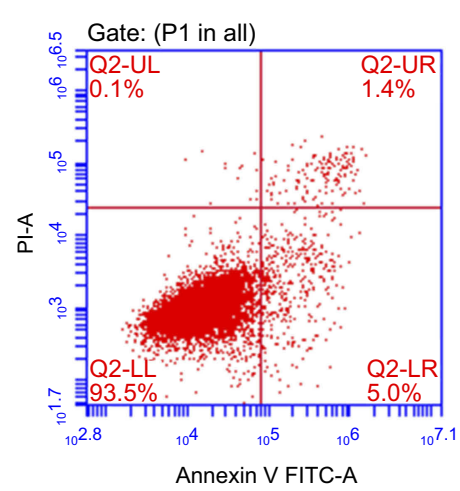

B

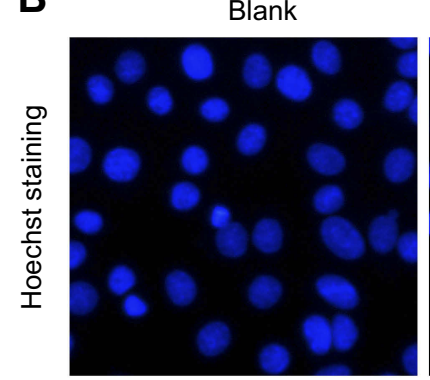

NC

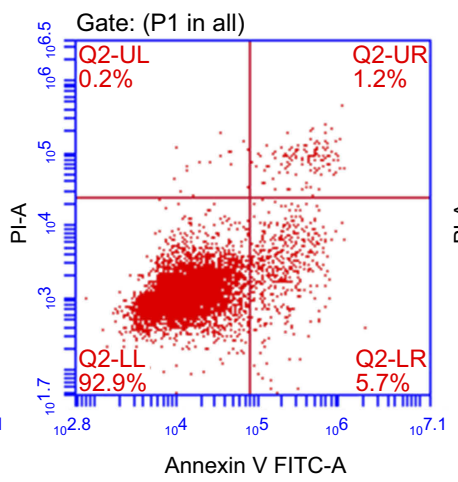

NC

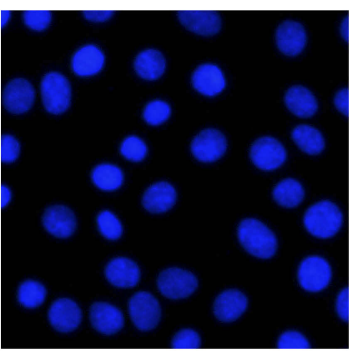

siRNA
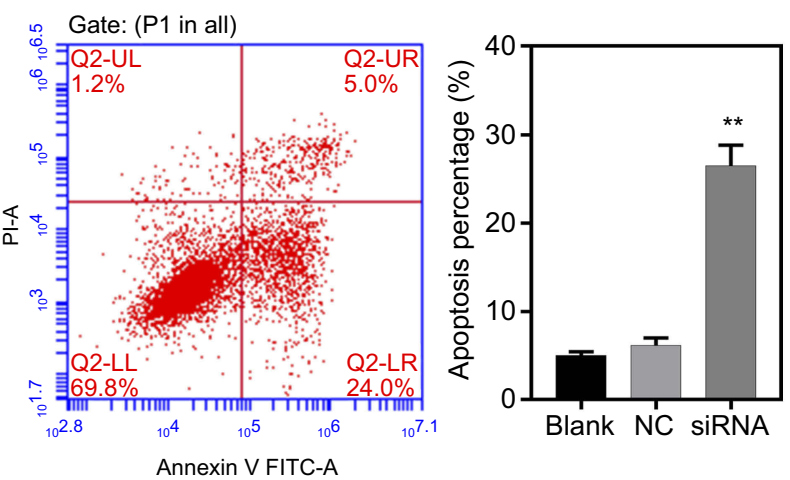

C

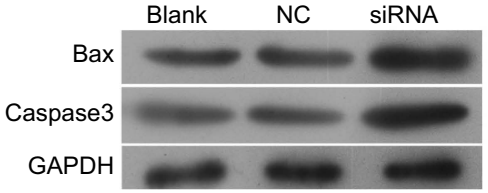

Figure 3 Knockdown of circLMNBI accelerated the apoptosis of LoVo cells. The apoptosis of LoVo cells with circLMNBI knockdown was assessed by flow cytometry (A) and Hoechst staining (B). (C) Knockdown of circLMNBI induced upregulation of Bax and caspase-3. **P<0.0I vs NC group.
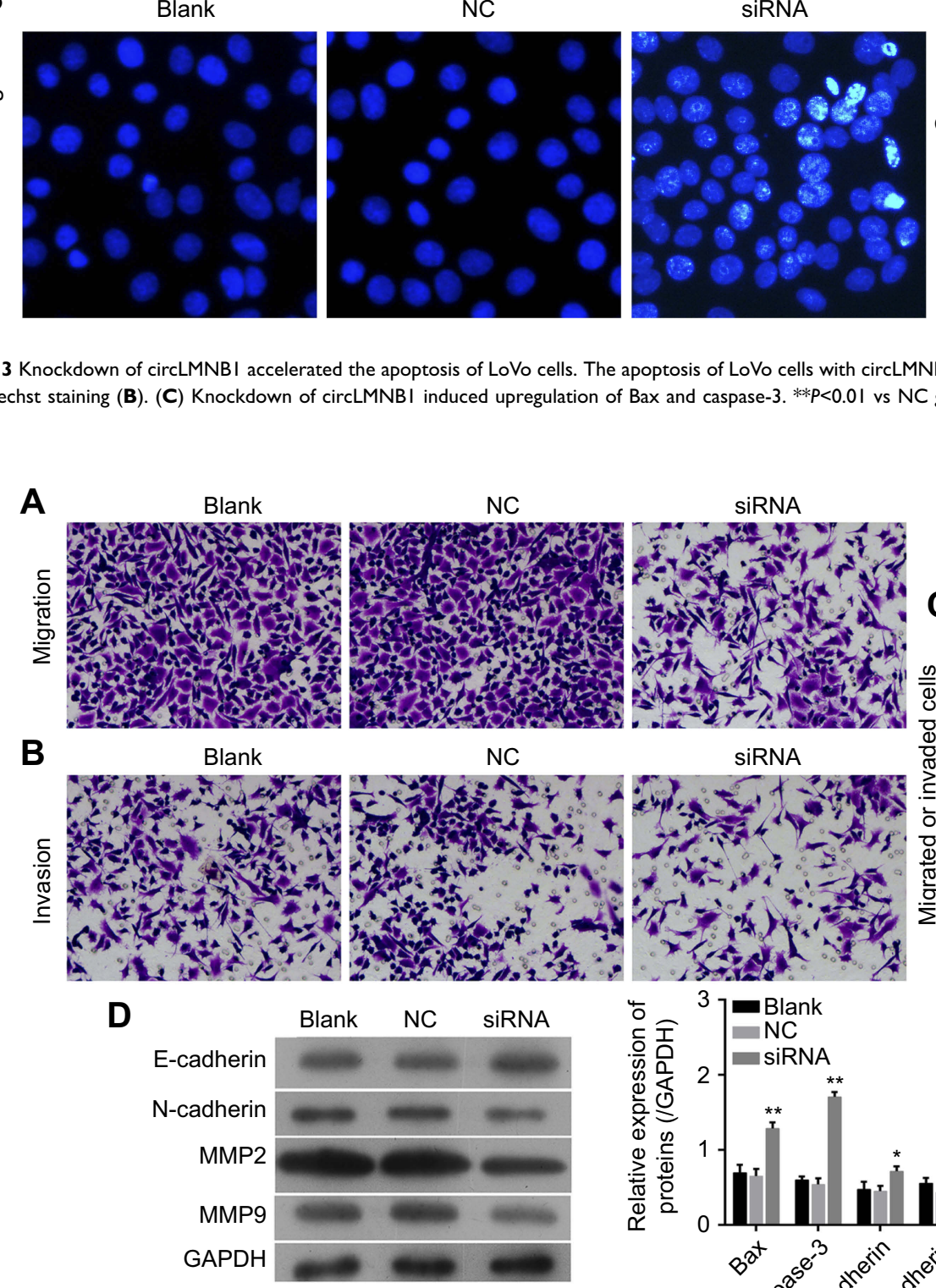

C
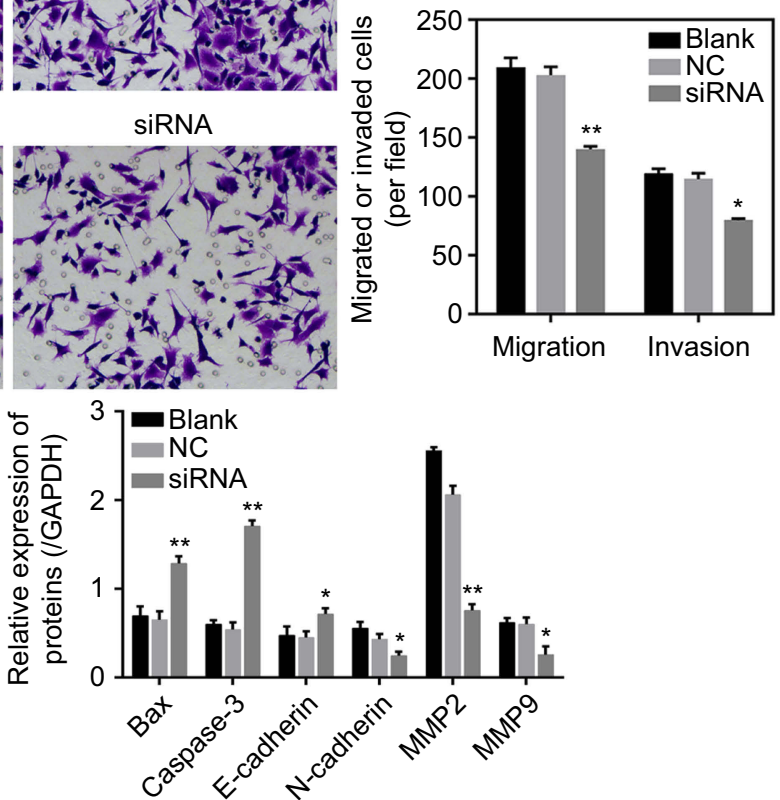

Figure 4 Knockdown of circLMNBI suppressed the EMT of LoVo cells. (A, B) Transwell assays were performed to detect the migration and invasion of LoVo cells with circLMLBI knockdown. (C) Expression of E-cadherin, N-cadherin, MMP2/9 were detected using Western blot. $* P<0.05$, $* * P<0.01$ vs NC group. 
(lncRNAs), are altered in many cancers, and aberrantly expressed miRNAs and (or) lncRNAs are involved in the epigenetic regulation of cancer development. ${ }^{18-21}$ Studies have also verified that most ncRNAs act as regulators in CRC development. ${ }^{2}$ However, only a few studies have sought to determine whether circRNAs have a significant effect in CRC. CircRNAs are constructed by the exons of various transcription genes found in human cells, suggesting that circRNAs are generated by abnormal splicing, non-linear reverse splicing, or gene rearrangement. ${ }^{22}$ In addition, when compared with miRNAs and lncRNAs, circRNAs are characterized by their sequence conservation, tissue specificity, and biological stability. Therefore, circRNAs are considered as possible biomarkers and therapeutic targets, and are thought to play roles in regulating gene expression. ${ }^{23-25}$ Recent studies have shown that circRNAs are involved in the development and progression of many cancers. For example, circ_0026344 was found to help suppress CRC progression via miRNA-21 and miRNA-31; ${ }^{26}$ circRNA_001569 was shown to participate in the proliferation and invasion of CRC cells by targeting miR-145 ${ }^{27}$ circRNA_100290 was identified as a prognostic biomarker that promotes CRC progression by regulating miR-516b and $\mathrm{Wnt} /$ beta-catenin signaling. ${ }^{28} \mathrm{In}$ our study, we proved for the first time that circLMNB1 is highly expressed in CRC tissues.

Cell proliferation plays a key role in the development of cancer. ${ }^{29}$ At the same time, cell proliferation and apoptosis occur in a state of dynamic equilibrium under physiological conditions. Once this balance is disrupted, inappropriate apoptosis might lead to a variety of diseases, such as cancers. ${ }^{29}$ Apoptosis is a protease cascade reaction mediated by members of the caspase family. ${ }^{30}$ When exposed to apoptosis-inducing signals, caspases become activated by binding to specific cofactors. Next, the activated caspases hydrolyze various proteins, and thereby activate additional responsive downstream caspases. After the responsive caspases become activated, they hydrolyze their targeted substances in cells, and cellular proteins are degraded, which eventually leads to irreversible cell death. ${ }^{31}$ Caspase-3 is the gene mostly commonly expressed in downstream apoptotic pathways, and plays a central role in the apoptosis process. ${ }^{32}$ Bax is a pro-apoptotic protein that activates caspase-3 and initiates the caspase cascade. ${ }^{33}$ In our study, we demonstrated that circLMNB1 knockdown inhibited proliferation, and promoted cell cycle arrest in LoVo cells. In addition, we proved that circLMNB1 knockdown facilitated LoVo cell apoptosis, and upregulated Bax and caspase- 3 expression.

Invasion and metastasis are two of the most important features of cancer cells. ${ }^{34}$ The EMT is a process by which epithelial cells are transformed into mesenchymal states, which promotes the metastasis of multifarious tumor cells. ${ }^{35}$ Cadherin switching is an important mechanism of the EMT. The transformation of E-cadherin into $\mathrm{N}$-cadherin is a potential mechanism that allows for tumor progression and metastasis. $^{36}$ In our study, we demonstrated that knockdown of circLMNB1 upregulated E-cadherin expression, and downregulated $\mathrm{N}$-cadherin expression in LoVo cells, suggesting that knockdown of circLMNB1 inhibited the EMT. Additionally, the EMT is always accompanied by an upregulation of matrix metalloproteinases (MMPs). ${ }^{37}$ The degradation of extracellular matrix (ECM) and basement membrane is the main pathological process that allows tumor cells leave a primary lesion and infiltrate adjacent tissue. $^{38}$ Matrix metalloproteinases (MMPs) are responsible for the degradation of numerous ECM components. ${ }^{39}$ MMPs can be divided into $>20$ categories based on their specific structural domains. MMP2 and MMP9 are closely associated with tumor dissemination and invasiveness, because they are known to degrade various gelatin substrates and type IV collagen. ${ }^{40} \mathrm{~A}$ substantial body of research has shown that ADAM17 can promote prostate cancer cell invasion by targeting MMP2 and MMP $9,{ }^{41}$ overexpression of derlin-1 promotes non-small cell lung cancer invasion by upregulating MMP2 and MMP$9,{ }^{42}$ and that MMP2 and MMP9 expression are associated with the metastatic phenotype of lung adenocarcinoma. $^{43}$ In this study, knockdown of circLMNB1 downregulated MMP2 and MMP-9 expression in LoVo cells, further suggesting that knockdown of circLMNB1 could suppress CRC metastasis.

In summary, circLMNT1 expression was demonstrated to be upregulated in CRC tissues when compared with its expression in para-carcinoma tissues. In vitro studies showed that knockdown of circLMNB1 inhibited cell proliferation, migration, and invasion, and promoted cell cycle arrest and apoptosis in LoVo cells. This study is the first to suggest circLMNB1 as a potential therapeutic target for CRC patients. 
A

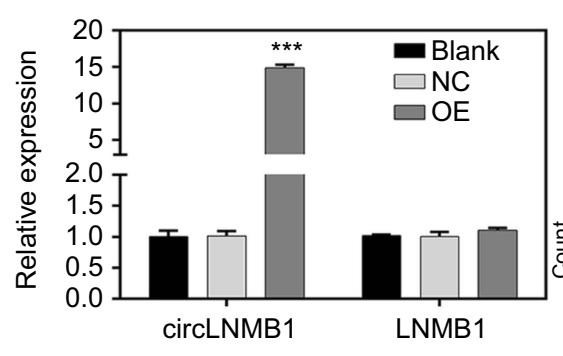

B

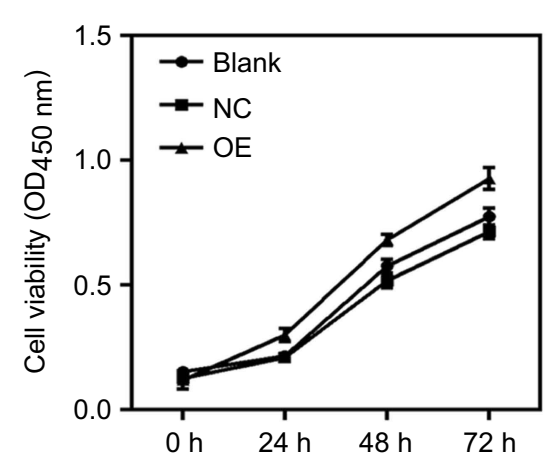

E

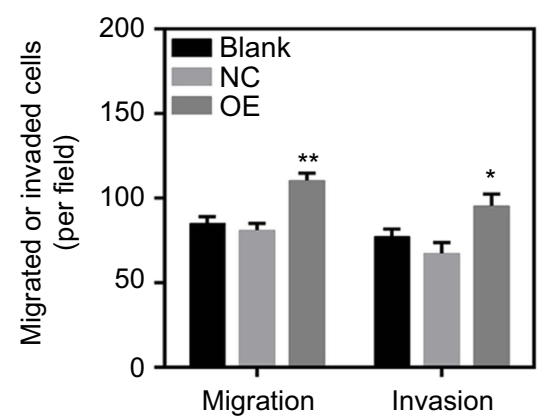

G

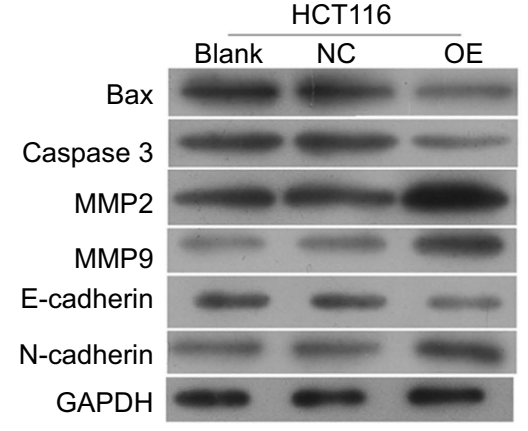

Blank

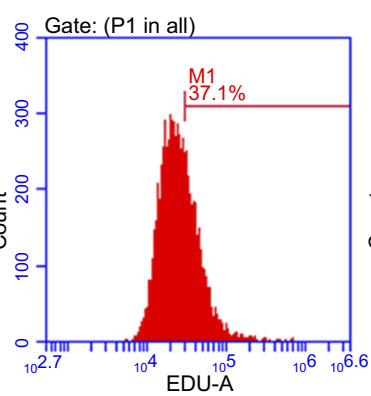

D
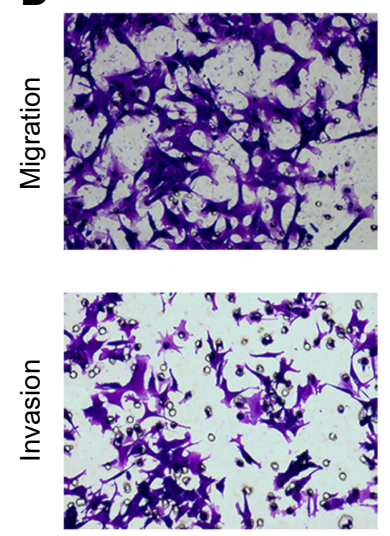

$\mathbf{F}$

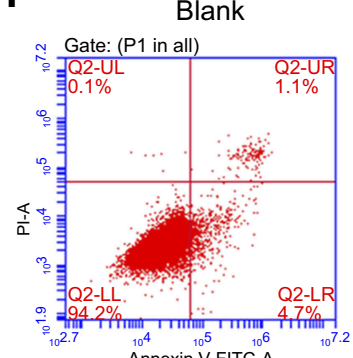

NC

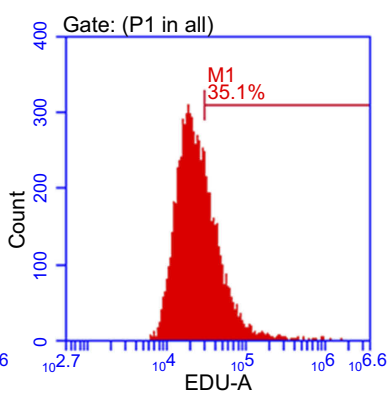

NC
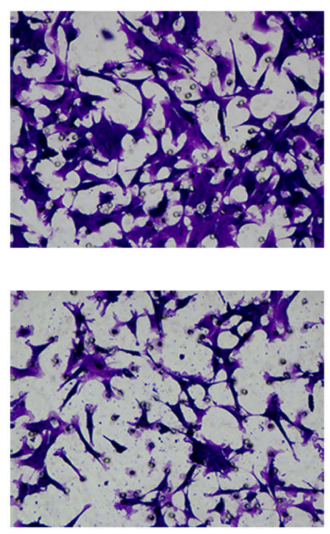

NC

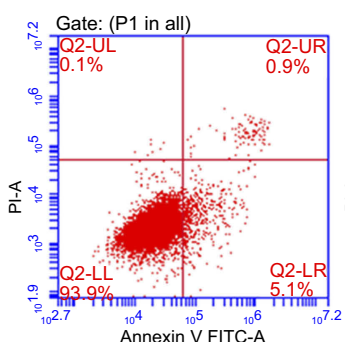

OE

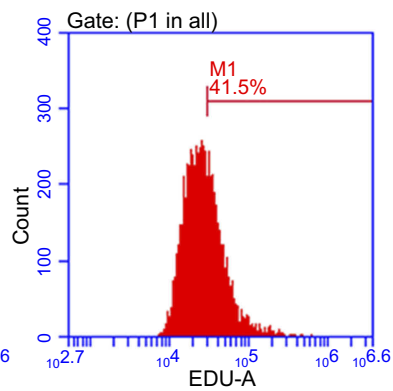

OE
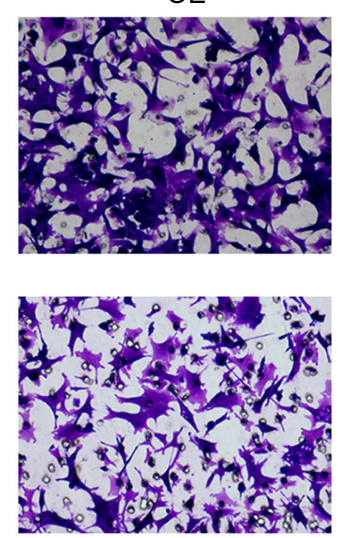

OE
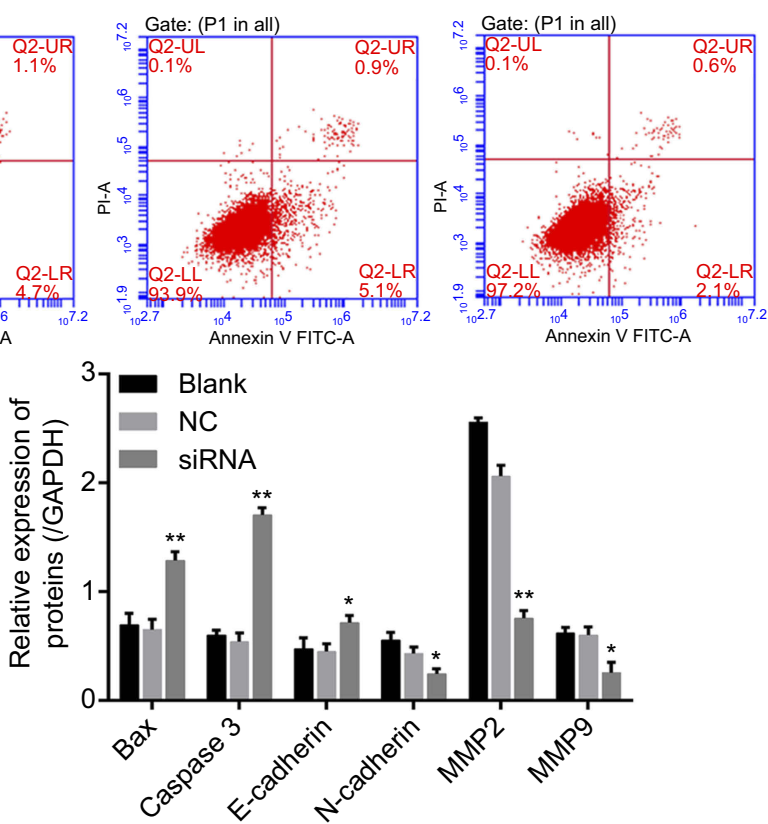

Figure 5 Overexpression of circLMNBI increased the malignant characteristics of cancer cells. (A) CircLMNBI and LMNBI expression were detected by qPCR. (B, C) Cell viability (B) and proliferation (C) were detected by CCK8 and EdU staining. (D, E) Cell migration and invasion were enhanced by circLMNBI. (F) Cell apoptosis was inhibited by circLMNBI. (G) The expression of apoptosis- and metastasis-related proteins was mediated by circLMNBI. *** indicate $P<0.05$ and $P<0.0$ I vs NC, respectively. 


\section{Disclosure}

The authors report no conflicts of interest in this work.

\section{References}

1. Song M, Garrett WS, Chan AT. Nutrients, foods, and colorectal cancer prevention. Gastroenterology. 2015;148(6):1244-1260. e1216. doi:10.1053/j.gastro.2014.12.035

2. Yiu AJ, Yiu CY. Biomarkers in colorectal cancer. Anticancer Res. 2016;36(3):1093-1102.

3. Chen LL, Yang L. Regulation of circRNA biogenesis. RNA Biol. 2015;12(4):381-388. doi:10.1080/15476286.2015.1020271

4. Guo JU, Agarwal V, Guo H, Bartel DP. Expanded identification and characterization of mammalian circular RNAs. Genome Biol. 2014;15(7):409. doi:10.1186/s13059-014-0409-z

5. Barrett SP, Wang PL, Salzman J. Circular RNA biogenesis can proceed through an exon-containing lariat precursor. Elife. 2015;4: e07540. doi:10.7554/eLife.06416

6. Wang PL, Bao Y, Yee MC, et al. Circular RNA is expressed across the eukaryotic tree of life. PLoS One. 2014;9(6):e90859. doi:10.1371/ journal.pone.0090859

7. Yuan G, Chen T, Zhang H, et al. Comprehensive analysis of differential circular RNA expression in a mouse model of colitis-induced colon carcinoma. Mol Carcinog. 2018. doi:10.1002/mc.22900

8. Shi P, Sun J, He B, et al. Profiles of differentially expressed circRNAs in esophageal and breast cancer. Cancer Manag Res. 2018;10:2207-2221. doi:10.2147/CMAR.S167863

9. Cheng XY, Shen H. [Circular RNA in lung cancer research: biogenesis, functions and roles]. Zhongguo Fei Ai Za Zhi. 2018;21(1):5056. doi:10.3779/j.issn.1009-3419.2018.01.07

10. Jiang W, Zhang X, Chu Q, et al. The circular RNA profiles of colorectal tumor metastatic cells. Front Genet. 2018;9:34. doi:10.3389/fgene.2018.00173

11. Wang P, He X. Current research on circular RNAs associated with colorectal cancer. Scand J Gastroenterol. 2017;52(11):1203-1210. doi:10.1080/00365521.2017.1365168

12. Zhang P, Zuo Z, Shang W, et al. Identification of differentially expressed circular RNAs in human colorectal cancer. Tumour Biol. 2017;39(3):1010428317694546.

13. Quail DF, Joyce JA. Microenvironmental regulation of tumor progression and metastasis. Nat Med. 2013;19(11):1423-1437. doi:10.1038/nm.3394

14. Song W, Mazzieri R, Yang T, Gobe GC. Translational significance for tumor metastasis of tumor-associated macrophages and epithelialmesenchymal transition. Front Immunol. 2017;8:1106. doi:10.3389/ fimmu.2017.01106

15. Smith BN, Bhowmick NA. Role of EMT in metastasis and therapy resistance. J Clin Med. 2016;5:2. doi:10.3390/jcm5020017

16. Santamaria PG, Moreno-Bueno G, Portillo F, Cano A. EMT: present and future in clinical oncology. Mol Oncol. 2017;11(7):718-738. doi:10.1002/1878-0261.12091

17. Livak KJ, Schmittgen TD. Analysis of relative gene expression data using real-time quantitative PCR and the 2(-Delta Delta C(T)) method. Methods (San Diego, CA). 2001;25(4):402-408. doi:10.1006/meth.2001.1262

18. Chen QN, Wei CC, Wang ZX, Sun M. Long non-coding RNAs in anti-cancer drug resistance. Oncotarget. 2017;8(1):1925-1936. doi:10.18632/oncotarget.12461

19. Slaby O, Laga R, Sedlacek O. Therapeutic targeting of non-coding RNAs in cancer. Biochem J. 2017;474(24):4219-4251. doi:10.1042/ BCJ20170079

20. Acunzo M, Romano G, Wernicke D, Croce CM. MicroRNA and cancera brief overview. Adv Biol Regul. 2015;57:1-9. doi:10.1016/j. jbior.2014.09.013
21. Rupaimoole R, Slack FJ. MicroRNA therapeutics: towards a new era for the management of cancer and other diseases. Nat Rev Drug Discov. 2017;16(3):203-222. doi:10.1038/nrd.2016.246

22. Salzman J, Gawad C, Wang PL, Lacayo N, Brown PO. Circular RNAs are the predominant transcript isoform from hundreds of human genes in diverse cell types. PLoS One. 2012;7(2):e30733. doi:10.1371/journal.pone.0030733

23. Li P, Chen S, Chen H, et al. Using circular RNA as a novel type of biomarker in the screening of gastric cancer. Clin Chim Acta. 2015;444:132-136. doi:10.1016/j.cca.2015.02.018

24. Memczak S, Jens M, Elefsinioti A, et al. Circular RNAs are a large class of animal RNAs with regulatory potency. Nature. 2013;495 (7441):333-338. doi:10.1038/nature11928

25. Jeck WR, Sorrentino JA, Wang K, et al. Circular RNAs are abundant, conserved, and associated with ALU repeats. RNA (New York, NY). 2013;19(2):141-157. doi:10.1261/rna.035667.112

26. Yuan Y, Liu W, Zhang Y, Zhang Y, Sun S. CircRNA circ_0026344 as a prognostic biomarker suppresses colorectal cancer progression via microRNA-21 and microRNA-31. Biochem Biophys Res Commun. 2018;503(2):870-875. doi:10.1016/j.bbrc.2018.06.089

27. Xie H, Ren X, Xin S, et al. Emerging roles of circRNA_001569 targeting miR-145 in the proliferation and invasion of colorectal cancer. Oncotarget. 2016;7(18):26680-26691. doi:10.18632/oncotarget.8589

28. Fang G, Ye BL, Hu BR, Ruan XJ, Shi YX. CircRNA_100290 promotes colorectal cancer progression through miR-516b-induced downregulation of FZD4 expression and Wnt/beta-catenin signaling. Biochem Biophys Res Commun. 2018;504(1):184-189. doi:10.1016/j. bbrc.2018.08.152

29. Evan GI, Vousden KH. Proliferation, cell cycle and apoptosis in cancer. Nature. 2001;411(6835):342-348. doi:10.1038/35077213

30. Goldar S, Khaniani MS, Derakhshan SM, Baradaran B. Molecular mechanisms of apoptosis and roles in cancer development and treatment. Asian Pac J Cancer Prev. 2015;16(6):2129-2144. doi:10.7314/ apjcp.2015.16.6.2129

31. Julien O, Wells JA. Caspases and their substrates. Cell Death Differ. 2017;24(8):1380-1389. doi:10.1038/cdd.2017.44

32. Porter AG, Janicke RU. Emerging roles of caspase-3 in apoptosis. Cell Death Differ. 1999;6(2):99-104. doi:10.1038/sj.cdd.4400476

33. Zheng JH, Viacava Follis A, Kriwacki RW, Moldoveanu T. Discoveries and controversies in BCL-2 protein-mediated apoptosis. FEBS J. 2016;283(14):2690-2700. doi:10.1111/febs.13527

34. Gupta GP, Massague J. Cancer metastasis: building a framework. Cell. 2006;127(4):679-695. doi:10.1016/j.cell.2006.11.001

35. Wang D, Li YJ, Ding N, et al. [Molecular networks and mechanisms of epithelial-mesenchymal transition regulated by miRNAs in the malignant melanoma cell line]. Yi Chuan. 2015;37(7):673-682. doi:10.16288/j.yczz.15-022

36. van Roy F. Beyond E-cadherin: roles of other cadherin superfamily members in cancer. Nat Rev Cancer. 2014;14(2):121-134. doi:10.1038/ $\operatorname{nrc3647}$

37. Vos MC, Hollemans E, Ezendam N, et al. MMP-14 and CD44 in Epithelial-to-Mesenchymal Transition (EMT) in ovarian cancer. $J$ Ovarian Res. 2016;9(1):53. doi:10.1186/s13048-016-0262-7

38. Jablonska-Trypuc A, Matejczyk M, Rosochacki S. Matrix metalloproteinases (MMPs), the main extracellular matrix (ECM) enzymes in collagen degradation, as a target for anticancer drugs. J Enzyme Inhib Med Chem. 2016;31(sup1):177-183. doi:10.3109/14756366.2016.1161620

39. Yadav L, Puri N, Rastogi V, Satpute P, Ahmad R, Kaur G. Matrix metalloproteinases and cancer - roles in threat and therapy. Asian Pac $J$ Cancer Prev. 2014;15(3):1085-1091. doi:10.7314/apjcp.2014.15.3.1085

40. Shay G, Lynch CC, Fingleton B. Moving targets: emerging roles for MMPs in cancer progression and metastasis. Matrix Biol. 2015;4446:200-206. doi:10.1016/j.matbio.2015.01.019 
41. Xiao LJ, Lin P, Lin F, et al. ADAM17 targets MMP-2 and MMP-9 via EGFR-MEK-ERK pathway activation to promote prostate cancer cell invasion. Int J Oncol. 2012;40(5):1714-1724. doi:10.3892/ijo.2011.1320

42. Dong QZ, Wang Y, Tang ZP, et al. Derlin-1 is overexpressed in nonsmall cell lung cancer and promotes cancer cell invasion via EGFRERK-mediated up-regulation of MMP-2 and MMP-9. Am J Pathol. 2013;182(3):954-964. doi:10.1016/j.ajpath.2012.11.019
43. Cai X, Zhu H, Li Y. PKCzeta, MMP2 and MMP9 expression in lung adenocarcinoma and association with a metastatic phenotype. Mol Med Rep. 2017;16(6):8301-8306. doi:10.3892/mmr.2017.7634

\section{Publish your work in this journal}

OncoTargets and Therapy is an international, peer-reviewed, open access journal focusing on the pathological basis of all cancers, potential targets for therapy and treatment protocols employed to improve the management of cancer patients. The journal also focuses on the impact of management programs and new therapeutic

Submit your manuscript here: https://www.dovepress.com/oncotargets-and-therapy-journal agents and protocols on patient perspectives such as quality of life, adherence and satisfaction. The manuscript management system is completely online and includes a very quick and fair peer-review system, which is all easy to use. Visit http://www.dovepress.com/ testimonials.php to read real quotes from published authors. 